\title{
Clinicopathologic and prognostic significance of LGR5, a cancer stem cell marker in patients with colorectal cancer
}

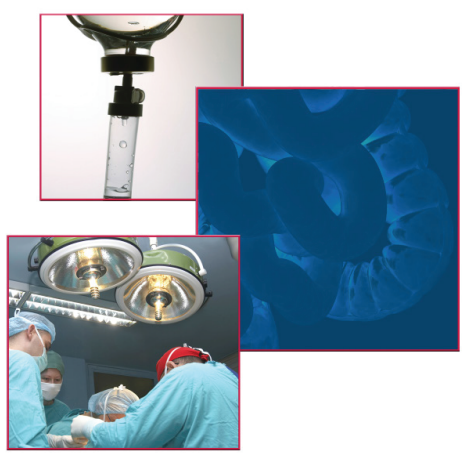

\author{
Chukwuemeka Ihemelandu*,1,2, Aisha Naeem ${ }^{2,3}$, Erika Parasido², Deborah Berry, 4, \\ Krysta Chaldekas 4,5 , Brent T Harris ${ }^{4,6}$, Olga Rodriguez ${ }^{2,5}$ \& Christopher Albanese ${ }^{2,5}$ \\ ${ }_{1}^{1}$ Program in Peritoneal Surface Oncology, MedStar Surgical Oncology, Department of Surgery, MedStar Georgetown University \\ Hospital, 3800 Reservoir Rd, NW Washington, DC 20007, USA \\ ${ }^{2}$ Preclinical Imaging Research Laboratory, Center for Cell Reprogramming, Department of Oncology and Pathology, Lombardi \\ Comprehensive Cancer Center, Georgetown University Medical Center, 3800 Reservoir Rd, NW Washington, DC 20007, USA \\ ${ }^{3}$ Biostatistician, Preclinical imaging Research Laboratory, Department of Oncology and Pathology, Lombardi Comprehensive Cancer \\ Center, Georgetown University Medical center, 3800 Reservoir Rd, NW Washington, DC 20007, USA \\ ${ }^{4}$ Histopathology \& Tissue Shared Resource, Department of Oncology, Lombardi Comprehensive Cancer Center, Georgetown \\ University Medical Center, 3900 Reservoir Rd, Washington, DC 20007, USA \\ ${ }^{5}$ Department of Oncology, Lombardi Comprehensive Cancer Center, Georgetown University Medical Center \\ ${ }^{6}$ Departments of Neurology \& Pathology, Georgetown University Medical Center \\ *Author for correspondence: Tel.: +1 202877 4599; Fax: +1 202877 0448; ci86@georgetown.edu
}

\section{Practice points}

- Leucine-rich repeat-containing G-protein-coupled receptor 5 (LGR5) is a colorectal cancer (CRC) stem cell marker.

- LGR5 protein expression was noted to be higher in CRC samples than in noncancerous tissue.

- Our study noted a trend of an increase in the expression of LGR5 as we progress from normal through stage III, with a subtle drop off noted as we progress to stage IV.

- Actuarial analysis using the Kaplan-Meier yielded LGR5 expression, as a potential prognostic variable.

- The LGR5 (high) cohort had a median overall survival time of 9 years compared with 6.3 years for the LGR5 (low) cohort $(p=0.01)$.

- CRC patients overexpressing LGR5 exhibited a better clinical overall survival $(p=0.028)$.

- LGR5's prognostic significance was more pronounced in stage II CRC patients than in stage I, III or IV CRC patients.

- LGR5 was noted to have potential prognostic values for prognostication in this cohort of patients, with LGR5 overexpression noted to be an independent prognostic variable for an improved overall survival outcome.

- LGR5 is an independent prognostic marker for better clinical outcomes in CRC patients and might be used as a potential therapeutic target in CRCs.

Aim: Toanalyze the clinicopathologic and prognostic significance of Leucine-rich repeat-containing Gprotein-coupled receptor 5 (LGR5), a cancer stem cell marker expression in a cohort of colorectal cancer patients (CRC). Patients \& methods: A total of 76 formalin-fixed paraffin-embedded tissue blocks of primary or metastatic tumors from 49 CRC patients were collected for duration 2009-2015. LGR5 expression was assessed through immunohistochemical staining of a tissue microarray. Results: LGR5 was significantly over expressed in CRC tissue samples and found to be a statistically significant independent prognostic marker for an improved overall survival. Conclusion: LGR5 expression was higher in colorectal cancer than in normal tissue. LGR5 was an independent prognostic marker for better clinical outcomes and might be used as a potential therapeutic target in CRCs.

First draft submitted: 12 August 2019; Accepted for publication: 8 October 2019; Published online: 12 November 2019

Keywords: biomarkers $\bullet$ cancer stem cell marker $\bullet$ colorectal cancer $\bullet$ immunohistochemistry $\bullet$ Leucine-rich repeatcontaining G-protein-coupled receptor $5 \bullet$ LGR5 • metastatic • overall survival • tissue microarray • tumor stage

Colorectal cancer (CRC) ranks third among both males and females, in both incidence and cancer-related deaths, accounting for approximately $9 \%$ of all cancer-related deaths in the USA [1]. Approximately $4-19 \%$ of patients 
who are diagnosed with CRC will be found to have peritoneal metastasis (PM), following an index curative surgical intervention. Likewise, approximately $44 \%$ of patients undergoing surgery for a diagnosis of recurrent $\mathrm{CRC}$ will be found to have PM $[2,3]$. For CRC patients diagnosed with metastatic disease confined to the peritoneal cavity, a novel therapeutic approach, cytoreductive surgery in combination with hyperthermic intraperitoneal chemotherapy offers this cohort of patients a 5 -year survival of up to $40 \%$ when a complete cytoreduction is achieved [4]. Unfortunately, despite these advances in multimodal treatment including systemic therapy [5], peritoneal metastasis remains a significant problem, due in part to a lack of effective diagnostics and therapeutics, underpinned by gaps in our understanding of molecular drivers of cancer and its progression. Hence, novel and effective diagnostic and therapeutic approaches are urgently required to achieve long-term favorable clinical prognosis for CRC patients with PM.

A postulated theory behind CRC-PM recurrence following a presumed effective therapeutic intervention, is that of the cancer stem cells (CSCs), which are thought to be resistant to chemotherapeutic agents used for treatment and thus able to lead to tumor recurrence. These CSCs are therefore postulated to be the driving force behind cancer progression and metastasis, making them ideal therapeutic and diagnostic targets that can be identified by several specific markers including; Leucine-rich repeat-containing G-protein-coupled receptor 5 (LGR5). Ricci-Vitiani et al. documented that CRC originates from and is propagated by a small number of undifferentiated tumorigenic CRCs [6]. Barker et al. discovered that LGR5 is expressed in the crypt base of the small and large intestines and qualifies as the stem cell marker for cells with intestinal differentiation [7-9].

Onset and progression of CRC usually involves a dysregulation of the Wnt/ $\beta$-catenin signaling pathway, often activated by an $A P C$ gene mutation, a known negative regulator of the Wnt pathway [10,11]. LGR5 is found on Wnt/ $\beta$-catenin-dependent adult stem cells of the colon and regulates Wnt signaling to R-spondin receptors [12]. Unfortunately, the underlying mechanisms for the involvement of LGR5 in carcinogenesis are poorly understood. LGR5 overexpression has been associated with recurrence, metastasis and poor prognosis in CRC. Conversely, Ziskin et al. found no correlation with prognosis, concluding that LGR5 expression is not associated with a poor prognosis, as might be anticipated for a CSC marker [13]. It is obvious that the role of LGR5 in CRC progression, metastasis and patient survival remains controversial. Our goal was to analyze the clinicopathologic and prognostic significance of LGR5 expression in a cohort of CRC patients.

\section{Methods \& material}

Patients \& tissue specimens

Formalin-fixed paraffin-embedded (FFPE) tissue blocks of primary or metastatic tumors from 49 CRC patients were collected from MedStar Georgetown University Hospital for surgical events in the period 2009-2015. LGR5 expression was assessed at the protein level through immunohistochemical (IHC) staining of a tissue microarray (TMA) consisting of pairs of tumor tissue cores obtained from each of the FFPE blocks. Three CRC cohorts were identified for TMA construction and IHC staining: Group one: a total of 7 patients with paired but independent primary and distant metastasis surgical resection events; Group two: a total of 22 patients with distant metastatic resection ( \pm local tumor resection); and Group three: 20 patients with primary tumor resection (nonmetastatic). The metastatic lesions in the Group one cohort included tumor tissue from abdominal wall, liver, small intestine and kidney/ureter, while the metastatic lesions in the Group two cohort included tissue from liver, omentum, ovary, soft tissue and uterus. Across the TMA series, 60 total surgical events from the study cohort $(\mathrm{n}=49)$ were represented; 38 patients had single surgery and 11 patients had two surgical events.

The correlation between LGR5 expression and clinicopathologic parameters (gender, age at diagnosis, American Joint Committee on Cancer staging, lymph node status, histopathology retrieved from the patient's medical records and prognosis) was assessed by statistical analysis.

Research use of de-identified tissue specimen and data was approved under Institutional Review Board protocols 1992-048 and 2007-345 and through the Biospecimen Use Committee at Georgetown University Medical Center. The Reporting Recommendations for Tumor Marker Prognostic Studies (REMARK) [14] was used to report this study.

\section{Tissue microarray}

Archival FFPE tissue blocks of primary or metastatic CRC tumors and their respective normal tissues were identified from participants enrolled in the Indivumed biobank of Georgetown University Medical Center. Sections were cut for hematoxylin-eosin staining with regions assessed to be histopathologically representative of viable tumor, which 
were used for construction of a series of four TMA blocks. Paired tissue cores of $2.0 \mathrm{~mm}$ diameter were punched from each donor block and transferred into a recipient paraffin block. Tissue controls on the TMA included 20 noncancerous colon samples, 10 cell lines and 4 benign tissues (kidney, liver, prostate and testis). Cell line preparation included fixation followed by pelleting and subsequent re-suspension of the cells in HistoGel media in order to punch plugs of dispersed cells for the TMA series. A minimum of two cores per tissue and cell block were obtained, resulting in a total of 230 cores for analysis.

\section{Immunohistochemistry analysis}

IHC staining was performed on $45 \mu \mathrm{m}$ sections obtained from each TMA receiver block [15,16]; following this, these sections were deparaffinized and rehydrated. Next, endogenous peroxidase was blocked for $20 \mathrm{~min}$ in $3 \%$ hydrogen peroxide in water, following which the slides were treated for antigen retrieval in citrate buffer $(\mathrm{pH} 6)$ for $10 \mathrm{~min}$ at $95^{\circ} \mathrm{C}$ (DAKO PT Link, Glostrup, Denmark). The sections were then incubated for $1 \mathrm{~h}$ at room temperature with primary antibodies, anti-LGR5 antibody (clone OTI2A2 Origene, MD, USA). The optimal dilution for staining colon cancer tissue sections was optimized for the LGR5 antibody. After 30 min of incubation with DAKO envision containing horseradish peroxidase-conjugated goat anti-mouse secondary antibodies (K4001, Agilent, Glostrup, Denmark), the sections were visualized using a diaminobenzidine solution (DAB+; Agilent kit). The sections were counterstained with hematoxylin, dehydrated and mounted with Acrymount (Statlab, Mckinney, TX, USA). The entire slides were scanned for further analysis.

\section{Scoring}

The TMA sections were semiquantitatively scored for LGR5 staining by pathologist (Brent Harris) on an Olympus BX53 brightfield microscopy, Olympus corporation, MA, USA. Scanned images were also scored (Deborah Berry). The intensity and percentage of positive tumor cells within each core were scored independently and categorized. The intensity score of epithelial staining was defined as zero when there was no staining; one for weak staining; two for moderate staining; or three for strong staining. The TMA slide was scanned at $40 \times$ using Vectra 3.0 Automated Quantitative Pathology Imaging System, Akoya Biosciences, MA, USA. Ten fields were randomly selected from each slide for quantification. Automated quantification was performed using inForm software version 2.4.1 (Perkin Elmer, MA, USA). The percentage of cells stained was scored zero none, one low, two medium, three high. The immunoreactive score was used for the final scoring. Immunoreactive score gives a range of $0-12$ as a product of multiplication between positive cells proportion score (0-4) and staining intensity score (0-3) [17].

\section{Statistical analysis}

Descriptive statistics was used to assess the frequency distributions among the groups. Categorical variables between groups were compared with univariate analysis using chi-square statistics. Differences in group means were evaluated using one-way analysis of variance (ANOVA F test). Equality of survivorship for outcome measure overall survival as a function of the LGR5 groups was assessed using the log-rank test of Kaplan-Meier. The primary objective of the analysis was to identify factors associated with overall survival (OS). OS was defined as the time interval between the date of surgery and date of death or last follow-up. An actuarial analysis was performed initially, using the Kaplan-Meier method with two-tailed log-rank p-values to evaluate potential prognostic variables. Based on the univariate analyses, we constructed confounder models using the Cox proportional hazards model, to estimate the degree to which patient demographics and clinicopathologic tumor characteristics affected survival and the estimates from these models provided hazard ratios and 95\% CI adjusted for all variables in the model. Life tables were used to calculate 3- and 5-year OS rates. p-values were two-sided, with values of $<0.05$ considered as statistically significant. Statistical analyses were performed using SPSS 24.

\section{Results}

Of the 49 patients in our study cohort, 30 (61\%) were females versus 19 (39\%) males. Age at initial diagnosis ranged from 31.8 to 84.6 years, with a median age of 61.4 years. To assess the protein expression of LGR5 in CRCs, IHC staining of a TMA was performed on a series of primary or metastatic tumors, as well as benign colonic and noncolonic control tissues and, well-characterized cell lines. Upon analysis of individual cases, LGR5 protein expression was noted to be higher in CRC samples than in noncancerous tissue (Figure 1A).

We observed a high Spearman correlation between our pathologist's (Brent Harris) visual scores generated for individual TMA spots and our digital image analysis $(\mathrm{p}=0.00)$. We conducted a one-way ANOVA to evaluate 

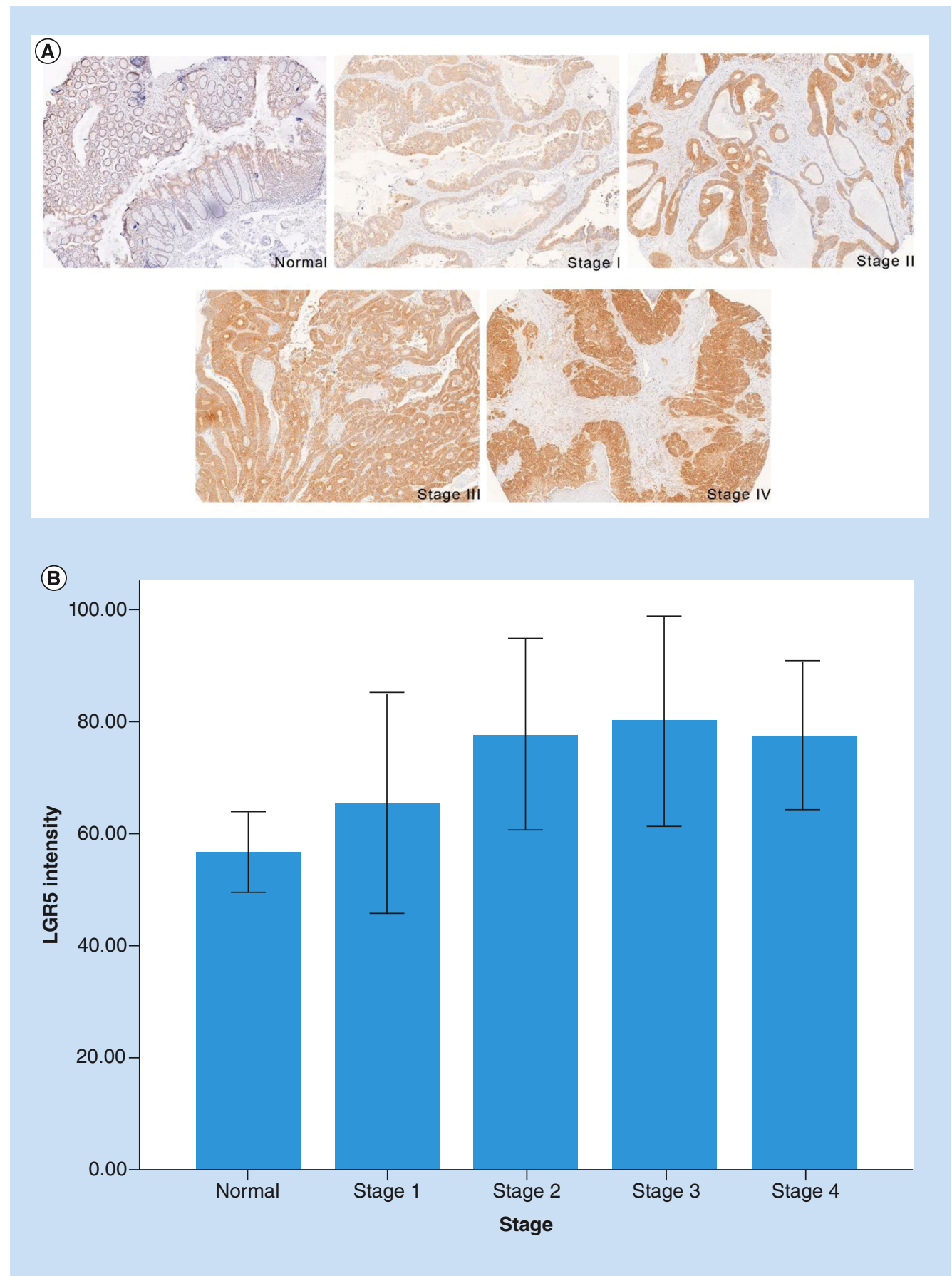

Figure 1. LGR5 expression in colon cancer. (A) Immunohistochemistry staining normal colon and stages I-IV colon cancer; representative images are shown. (B) Error bar graph, displaying the means and standard deviations for LGR5 staining intensity for each group; normal colon and stages I-IV colon cancer.

the relationship between tumor stage and LGR5 expression. The independent variable, the tumor stage factor, included normal tissue and stages I-IV colon cancer. The dependent variable was LGR5 expression. The ANOVA was significant, $p=0.05$. A trend is observed with an increase in the expression of LGR5 noted as we progress from normal through stage III, with a subtle drop off noted as we progress to stage IV. An error bar graph, displaying the means and standard deviations for LGR5 expression (staining intensity) for each group, normal colon and stages I-IV colon cancer is shown in (Figure 1B). 


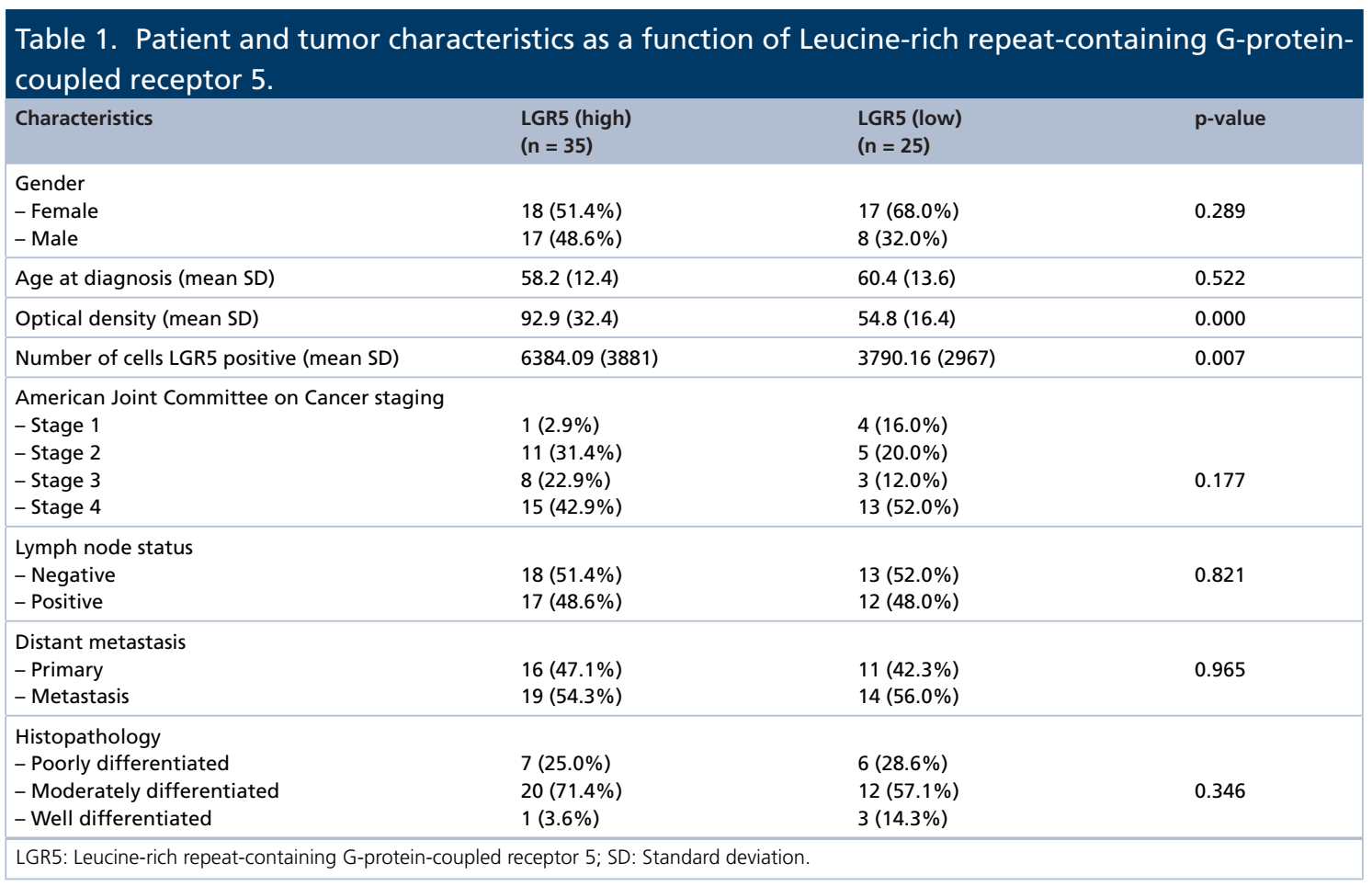

Table 2. Equality of survivorship for outcome measure overall survival as a function of Leucine-rich repeat-containing G-protein-coupled receptor 5 was assessed using the log-rank test of the KaplanMeier and life tables.

\begin{tabular}{|lll|}
\hline Characteristics & $\begin{array}{l}\text { LGR5 }(\mathbf{h i g h}) \\
(\mathbf{n}=\mathbf{3 5})\end{array}$ & $\begin{array}{l}\text { LGR5 (low) } \\
(\mathbf{n}=\mathbf{2 5})\end{array}$ \\
\hline Median follow-up time & 5.2 years $(1.8-9.3)$ & 5.0 years $(0.8-9.3)$ \\
\hline 3 years overall survival & $94 \%$ & $72 \%$ \\
\hline 5 years overall survival & $75 \%$ & $53 \%$ \\
\hline Median overall survival & 9.0 years & 6.3 years \\
\hline LGR5: Leucine-rich repeat-containing G-protein-coupled receptor 5. & \\
\hline
\end{tabular}

The clinicopathological characteristics of our study cohort as a function of LGR5 expression are presented in Table 1. No significant differences were observed between the cohorts of LGR5 (high) versus LGR5 (low), with regards to gender, age at diagnosis, American Joint Committee on Cancer staging, lymph node status, distant metastasis and histopathology. The mean optical density for the cohort of patients classified as LGR5 (high) was 92.9 versus 54.8 for the LGR5 (low) cohort $(\mathrm{p}=0.00)$.

\section{Survival analyses}

Duration of follow-up ranged from 0.8 to 9.3 years with a median of 5.2 years for the entire study cohort. Median follow-up time for the cohort LGR5 (high) was 5.2 versus 5.0 years for the LGR5 (low) cohort (Table 2). The LGR5 (high) cohort had a median OS time of 9 years compared with 6.3 years for the LGR5 (low) cohort $(\mathrm{p}=0.01)$. The 3- and 5-year OS rates were: 94, 75, 72 and 53\%, respectively, for the LGR5 (high) and LGR5 (low) cohorts (Table 2).

Actuarial analysis using the Kaplan-Meier yielded LGR5 expression as a potential prognostic variable Figure 2A. CRC patients overexpressing LGR5 exhibited a better clinical OS ( $p=0.028)$.

LGR5's prognostic significance was more pronounced in stage II CRC patients than in stage I, III or IV (Figure 2b).

Constructing a confounder model using Cox regression analysis and including all variables assessed in univariate analysis, LGR5 remarkably emerged as the only statistically significant independent predictor of OS on multivariate 


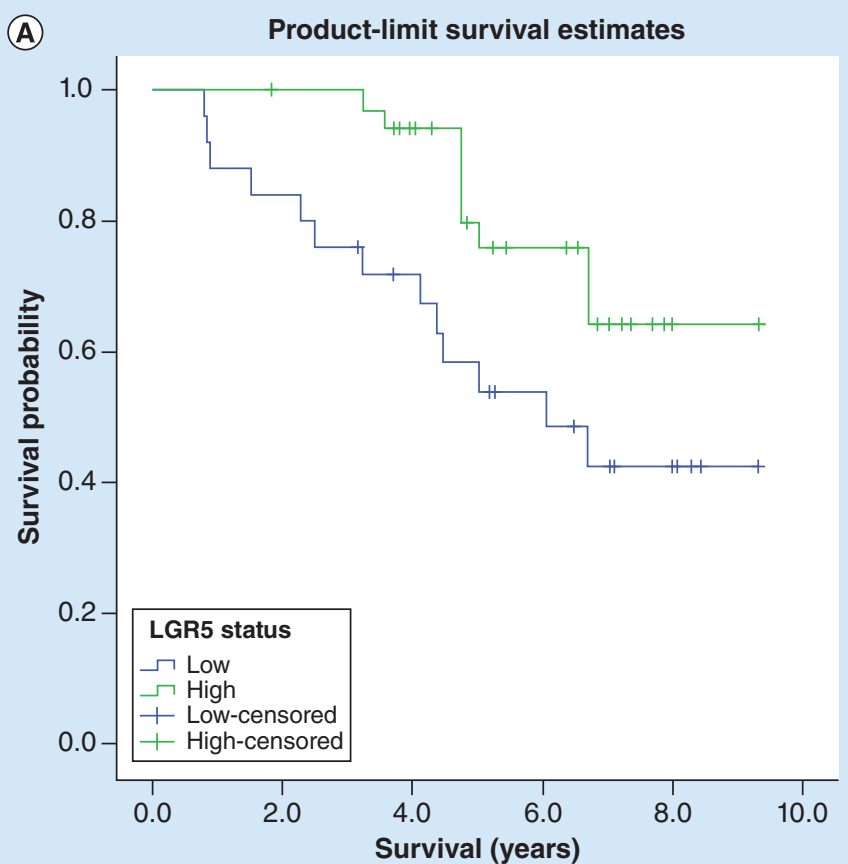

\section{(B) Product-limit survival estimates}

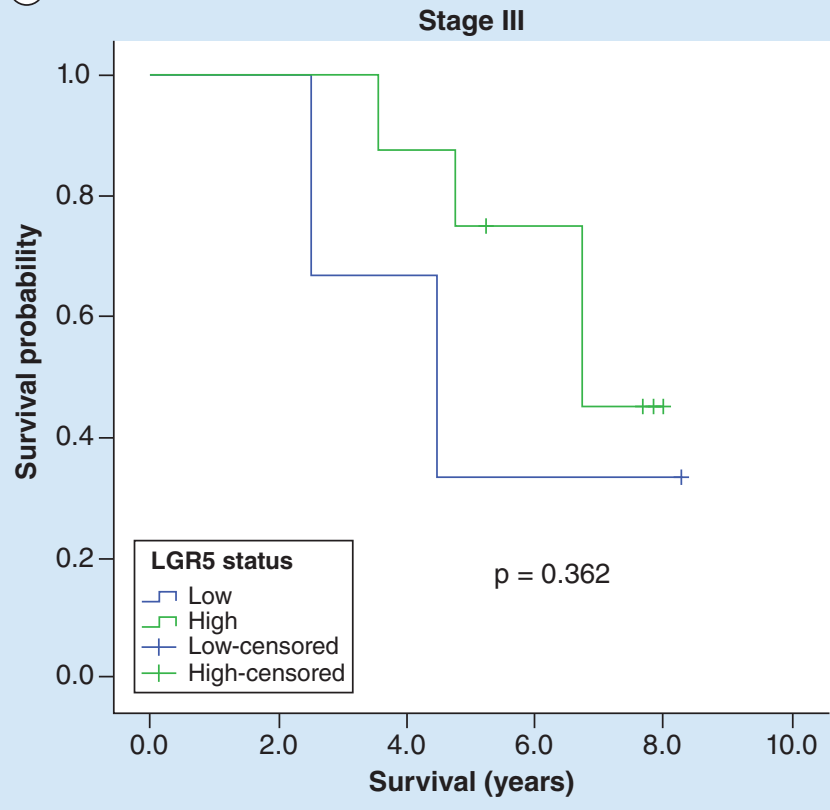

Product-limit survival estimates Stage I

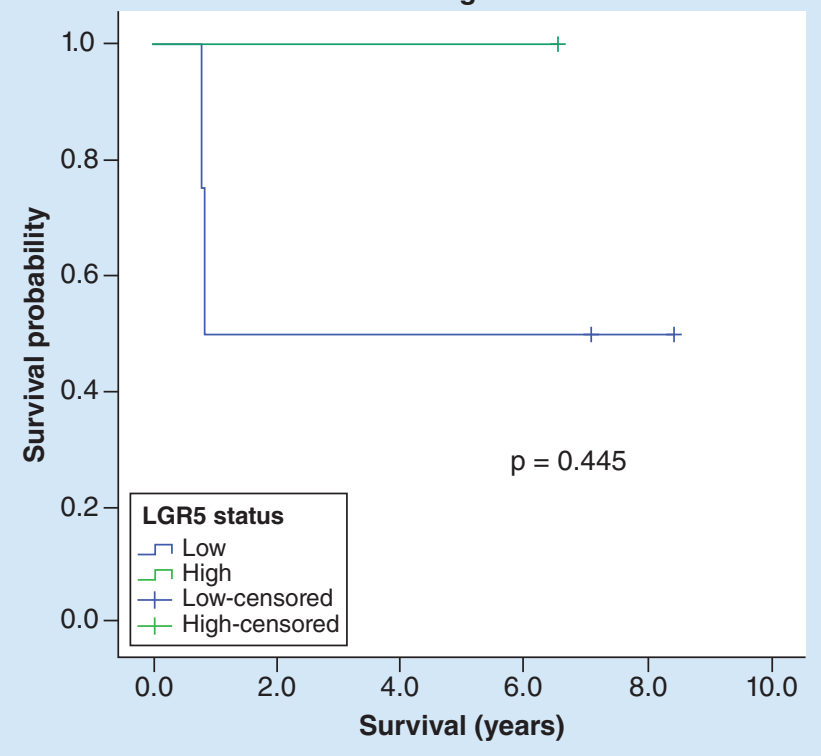

Figure 2. Univariate survival analysis of the entire cohort of patients with colorectal cancer as a function of Leucine-rich repeat-containing G-protein-coupled receptor 5 .

analysis (Table 3). Patients underexpressing LGR5 (low) (hazard ratio: 3.3; 95\% CI: 1.1-9.7; p = 0.029) were more likely to have a shorter survival when compared with patients overexpressing LGR5 (high).

\section{Discussion}

Our objective in this study was to analyze the clinicopathologic and prognostic significance of LGR5 expression in a cohort of CRC patients. Remarkably, our study shows LGR5 to have potential prognostic values for prognostication in this cohort of patients, with LGR 5 overexpression noted to be an independent prognostic variable for an improved 


\begin{tabular}{|c|c|c|}
\hline Characteristics & HR (95\% Cl) & $p$-value \\
\hline $\begin{array}{l}\text { Histopathology } \\
\text { - Poorly differentiated }{ }^{\dagger} \\
\text { - Moderately differentiated } \\
\text { - Well differentiated }\end{array}$ & $\begin{array}{l}1.0(0.2-4.0) \\
4.0(0.4-38.5)\end{array}$ & $\begin{array}{l}0.943 \\
0.230\end{array}$ \\
\hline $\begin{array}{l}\text { LGR5 status } \\
- \text { - High }^{\dagger} \\
\text { - Low }\end{array}$ & $3.3(1.1-9.7)$ & 0.029 \\
\hline $\begin{array}{l}\text { Distant metastasis } \\
\text { - Distant metastasis }{ }^{\dagger} \\
\text { - vs no distant metastasis }\end{array}$ & $0.7(0.1-3.5)$ & 0.678 \\
\hline $\begin{array}{l}\text { Gender } \\
\text { - Female }{ }^{\dagger} \\
\text { - vs male }\end{array}$ & $2.1(0.6-7.1)$ & 0.226 \\
\hline Age at diagnosis & $1.0(0.9-1.0)$ & 0.995 \\
\hline $\begin{array}{l}\text { Lymph node status } \\
\text { - Negative }{ }^{\dagger} \\
\text { - vs positive }\end{array}$ & $1.3(0.1-12.6)$ & 0.777 \\
\hline $\begin{array}{l}\text { American Joint Committee } 0 \\
\text { - Stage } 1^{\dagger} \\
\text { - Stage } 2 \\
\text { - Stage } 3 \\
\text { - Stage } 4\end{array}$ & $\begin{array}{l}0.8(0.1-7.2) \\
1.9(0.0-39.4) \\
0.9(0.0-15.2)\end{array}$ & $\begin{array}{l}0.901 \\
0.678 \\
0.949\end{array}$ \\
\hline
\end{tabular}

OS outcome. Our finding of the positive prognostic significance of LGR5 overexpression in a cohort of CRC patients confirms the findings of authors such as Jang et al. [18], Wu et al. [19] and runs contrary to the popular belief of CSC as the driving force behind cancer progression and metastasis. Our study using IHC staining of a TMA to evaluate the protein expression of LGR5, defines LGR5 as being overexpressed in CRC patients when compared with noncancerous colonic tissue and cancerous noncolonic tissue, hence making LGR5 a promising candidate for targeted therapy and diagnostics in CRC patients.

Also observed in our study was a trend toward an increase in the expression of LGR5 in the CRC tissues as we progressed from normal through stage III, with a subtle drop off noted as we progressed to stage IV. A similar trend was reported by Zhou et al. [20], who documented that, although there were overlaps in LGR5 expression between the different CRC stages, the average density of LGR5 staining was higher in the earlier stages I and II tumors when compared with normal crypts and were lower in the later stages III and IV tumors when compared with stages I and II tumors. Zhou et al. theorize that these results show that expression of LGR5 decreases in advanced stages of colon cancer as compared with the early stage disease, indicating that there may be an active selection for LGR5 expression early in CRC progression, or that this phenomenon may simply reflect the origin of the tumors as derived by clonal expansion of LGR5-positive stem cells, with suppression thereafter following tumor progression.

Jang et al. [18] studying the prognostic significance of LGR5 using RNA in situ hybridization in a large cohort of CRC samples also reported a similar finding, documenting that LGR5 expression was significantly lower in the metastatic cancer cells. Jang et al. also documented that LGR5 expression was much higher in CRC tissues than in noncancerous tissue and matched colon tissues $(\mathrm{p}<0.01)$ findings, which support our own results. Additionally, Jang et al. notably found LGR5 overexpression to be an independent prognostic marker associated with better survival. In an elegant study to explain this observed outcome, the authors document that overexpression of LGR5 impaired cancer progression by reducing ERK phosphorylation in addition to reducing the colony-forming and migration potentials in DLD1 cells. Also, knockdown of LGR5 expression in their study led to a reduction of the colony-forming and migration potentials in LoVo cells. Jang et al. conclude that their data suggest a suppressive role of LGR5 in CRC progression. Other recent publications have also documented a tumor-suppressive role of LGR5 signaling in human CRCs.

Wu et al. [19] studied RSPO2-LGR5 signaling and showed an inhibitory effect on cell growth mediated by a Wnt signaling-negative feedback loop, which is activated by RSPO2 acting as a tumor suppressor, through a RSPO2induced, LGR5-dependent pathway. Zhou et al. [20] states that their study uncovers a novel cross-talk between LGR5 and TGF $\beta$ signaling in colon cancer and identifies LGR5 as a new modulator of TGF $\beta$ signaling able to 
suppress colon cancer metastasis. Melo et al. [21] demonstrated that high expression levels of Wnt-targeted genes, which comprise ASCL2, LGR5, AXIN2 and APCDD1, are associated with a favorable prognosis in CRC patients. Xiang-Shan et al. documented that upregulation of LGR5 expression, especially in female patients, may play an important role in colorectal carcinogenesis, probably through the WNT/ $\beta$-catenin pathway, but no involvement in the progression of the CRC [22]. Other studies have documented LGR5 as having a negative prognostic influence in CRCs [23-25].

A review of the literature highlights the controversy swirling around the role of LGR5 in CRC progression, metastasis and more importantly patient survival, further emphasizing the gaps in our understanding of molecular drivers of CRC and its progression. Understanding and deciphering the true underlying mechanism associated with LGR5 inhibition or stimulation will be key to a successful translation of LGR5 as a diagnostic and therapeutic target. A Phase I, dose escalation study of an anti-LGR5 humanized monoclonal antibody in patients with metastatic CRC was recently terminated following the sponsor's decision (ClinicalTrials.gov identifier [NCT number]: NCT02726334). The stated aim of this anti-LGR5 was to prevent CRC recurrence and improve disease-free survival by targeting LRG5. The reasons for termination of this clinical trial have not been made known publicly. Most recently Nagata et al. [26] found that a negative LGR5 expression was a significant predictor of peritoneal recurrence in patients with pT4 colon cancer, while Sato et al. [27] documented that there was a significant difference in OS between the LGR5-positive group and LGR5-negative group, with a Cox proportional hazards models revealing the LGR5-positive group as having a better OS.

Other stem cell markers for CRC studied in the literature include CD44 and CD24, of which Sadeghi et al. [28] documented an inverse association with tumor aggressiveness for the epithelial isoform of CD44. Mirzaei et al. [29] elucidated the expression of doublecortin-like kinase 1 (DCLK1), another known stem cell marker in CRC and LGR5 expression, showing a higher mRNA expression level of both doublecortin-like kinase 1 and LGR5 in CRC samples, with a documented positive correlation between these two stem cell markers noted in their cohort of patients with CRC.

\section{Conclusion \& future perspective}

Our study validates the overexpression of LGR5 in CRC and supports its potential prognostic value.

This study has some important limitations, including the small cohort study size, its retrospective nature, which makes it difficult to control bias and confounders and, makes it difficult to establish cause and effect. Despite these limitations, our study underscores that LGR5 is a promising candidate for targeted therapy and diagnostics in CRC patients with peritoneal metastasis.

In conclusion, LGR5 expression was higher in CRC than in normal mucosa. LGR5 is an independent prognostic marker for better clinical outcomes in CRC patients and might be used as a potential therapeutic target in CRCs.

It is our belief that as the era of personalized medicine continues to evolve, and with the ever-increasing knowledge and use of targeted therapy for treatment of cancer, the need for ideal tumor protein targets is becoming ever more paramount. LGR5 appears to be an ideal tumor protein target for CRC, with its localization on the cell membrane, its diffuse upregulation throughout CRC tissue and its upregulation in the majority of CRC patients making it an ideal theranostic agent for CRC care.

Financial \& competing interests disclosure

Research reported in this publication was supported by the National Center For Advancing Translational Sciences of the NIH under award number KL2TR001432. The content is solely the responsibility of the authors and does not necessarily represent the official views of the NIH. The authors have no other relevant affiliations or financial involvement with any organization or entity with a financial interest in or financial conflict with the subject matter or materials discussed in the manuscript apart from those disclosed.

No writing assistance was utilized in the production of this manuscript.

Ethical conduct of research

The authors state that they have obtained appropriate institutional review board approval or have followed the principles outlined in the Declaration of Helsinki for all human or animal experimental investigations. In addition, for investigations involving human subjects, informed consent has been obtained from the participants involved. 


\section{Open access}

This work is licensed under the Attribution-NonCommercial-NoDerivatives 4.0 Unported License. To view a copy of this license, visit http://creativecommons.org/licenses/by-nc-nd/4.0/

\section{References}

Papers of special note have been highlighted as: $\bullet$ of interest; $\bullet \bullet$ of considerable interest

1. Siegel RL, Miller KD, Jemal A. Cancer statistics, 2017. CA Cancer J. Clin. 67(7), 7-9 (2017).

2. Jayne DG, Fook S, Loi C, Seow-Choen F. Peritoneal carcinomatosis from colorectal cancer. Br. J. Surg. 89(12), 1545-1550 (2002).

3. Koppe MJ, Boerman OC, Oyen WJG, Bleichrodt RP. Peritoneal carcinomatosis of colorectal origin - incidence and current treatment strategies. Ann. Surg. 243(2), 212-222 (2006).

4. Verwaal VJ, Bruin S, Boot $\mathrm{H}$ et al. 8-year follow-up of randomized trial: cytoreduction and hyperthermic intraperitoneal chemotherapy versus systemic chemotherapy in patients with peritoneal carcinomatosis of colorectal cancer. Ann. Surg. Onc. 15(9), 2426-2432 (2008).

5. National Cancer Institute. Drugs approved for colon and rectal cancer (2019). https://www.cancer.gov/about-cancer/treatment/drugs/colorectal

6. Ricci-Vitiani L, Lombardi DG, Pilozzi E et al. Identification and expansion of human colon-cancer-initiating cells. Nature 445(7123), 111-115 (2007).

7. Barker N, Van Es JH, Kuipers J et al. Identification of stem cells in small intestine and colon by marker gene LGR5. Nature 449, 1003-1007 (2007).

8. Barker $\mathrm{N}$, van Es JH, Jaks $\mathrm{V}$ et al. Very long-term self-renewal of small intestine, colon, and hair follicles from cycling LGR5+ve stem cells. Cold Spring Harb. Symp. Quant. Biol. 73, 351-356 (2008).

9. Montgomery RK, Breault DT. Small intestinal stem cell markers. J. Anat. 213(1), 52-58 (2008).

10. Morgan RG, Mortensson E, Williams AC. Targeting LGR5 in colorectal cancer: therapeutic gold or too plastic? Br. J. Cancer 118(11), 1410-1418 (2018).

11. Korinek V, Barker N, Morin PJ et al. Constitutive transcriptional activation by a $\beta$-catenin-Tcf complex in APC-/- colon carcinoma. Science 275(5307), 1784-1787 (1997).

12. de Lau W, Peng WC, Gros P et al. The R-spondin/LGR5/RNF43 module: regulator of Wnt signal strength. Genes Dev. 28(4), 305-316 (2014).

13. Ziskin JL, Dunlap D, Yaylaoglu M et al. In situ validation of an intestinal stem cell signature in colorectal cancer. Gut 62(7), 1012-1023 (2013).

14. Sauerbrei W, Taube SE, McShane LM et al. Reporting recommendations for tumor marker prognostic studies (REMARK): an abridged explanation and elaboration. J. Natl Cancer Inst. 110(8), 803-811 (2018).

15. Miller TJ, McCoy C, Hemmings MK et al. Objective analysis of cancer stem cell marker expression using immunohistochemistry. Pathology 49, 24-29 (2017).

16. Stammes MA, Prevoo HAJM, Ter Horst MC et al. Evaluation of EphA2 and EphB4 as targets for image-guided colorectal cancer surgery. Int. J. Mol. Sci. 18(2), 307 (2017).

17. Remmele W, Stegner HE. Recommendation for uniform definition of an immunoreactive score (IRS) for immunohistochemical estrogen receptor detection (ER-ICA) in breast cancer tissue. Pathol. 8(3), 138-140 (1987).

18. Jang BG, Kim HS, Chang WY et al. Expression profile of LGR5 and its prognostic significance in colorectal cancer progression. Am. J. Pathol. 188(10), 2236-2250 (2018).

-• Documents findings contrary to the popular belief of cancer stem cells as the driving force behind cancer progression and metastasis.

19. Wu C, Qiu S, Lu L et al. RSPO2-LGR5 signaling has tumour-suppressive activity in colorectal cancer. Nat. Commun. 5, 3149 (2014).

-. Documents findings contrary to the popular belief of cancer stem cells as the driving force behind cancer progression and metastasis.

20. Zhou X, Geng L, Wang D et al. R-Spondin1/LGR5 activates TGF $\beta$ signaling and suppresses colon cancer metastasis. Cancer Res. 77(23), 6589-6602 (2017).

-• Documents that, although there were overlaps in LGR5 expression between the different colorectal cancer stages, the average density of LGR5 staining was higher in the earlier stages I and II tumors when compared with normal crypts and were lower in the later stages III and IV tumors when compared with stages I and II tumors.

21. de Sousa E Melo F, Colak S, Buikhuisen J et al. Methylation of cancer-stem-cell-associated Wnt target genes predicts poor prognosis in colorectal cancer patients. Cell Stem Cell. 9(5), 476-485 (2011).

- Shows that high expression levels of Wnt-targeted genes, which comprise ASCL2, LGR5, AXIN2 and APCDD1, are associated with a favorable prognosis in colorectal cancer patients. 
22. Xiang-Shan F, Hong-Yan W, Hui-Ping Y et al. Expression of LGR5 in human colorectal carcinogenesis and its potential correlation with $\beta$-catenin. Int. J. Colorectal Dis. 25(5), 583-590 (2010).

- Documents that upregulation of LGR5 expression, especially in female patients, may play an important role in colorectal carcinogenesis, probably through the $\mathrm{WNT} / \beta$-catenin pathway, but does not involve the progression of the colorectal cancer.

23. He S, Zhou H, Zhu X et al. Expression of Lgr5, a marker of intestinal stem cells, in colorectal cancer and its clinicopathological significance. Biomed. Pharmacother. 68(5), 507-513 (2014).

- Documents LGR5 as having a negative prognostic influence in colorectal cancer.

24. Takahashi H, Ishii H, Nishida N et al. Significance of LGR5(+ve) cancer stem cells in the colon and rectum. Ann. Surg. Oncol. 18(4), 1166-1174 (2011).

25. Wu X-S, Xi H-Q, Chen L. LGR5 is a potential marker of colorectal carcinoma stem cells that correlates with patient survival. World J. Surg. Oncol. 10, 244 (2012).

26. Nagata $\mathrm{H}$, Ishihara $\mathrm{S}$, Abe $\mathrm{H}$ et al. LGR5 expression predicts peritoneal recurrence after curative resection of primary colon cancer. $B r$. J. Cancer 120(10), 996-1002 (2019).

-• Finds that a negative LGR5 expression is a significant predictor of peritoneal recurrence in patients with pT4 colon cancer.

27. Sato K, Uehara T, Iwaya M et al. Correlation of clinicopathological features and LGR5 expression in colon Adenocarcinoma. Ann. of Diagn. Pathol. 40, 161-165 (2019).

-• Documents that there is a significant difference in overall survival between the LGR5-positive group and LGR5-negative group with a Cox proportional hazards models revealing the LGR5-positive group as having a better overall survival.

28. Sadeghi A, Roudi R, Mirzaei A et al. CD44 epithelial isoform inversely associates with invasive characteristics of colorectal cancer. Biomark Med. 13(6), 419-426 (2019).

29. Mirzaei A, Tavoosidana G, Rad AA et al. A new insight into cancer stem cell markers: could local and circulating cancer stem cell markers correlate in colorectal cancer? Tumor Biol. 37(2), 2405-2414 (2016). 